Co-construction du « capital célébrité » du présidentiable en ligne. Analyse comparative des sites de campagne en 2012

Co-Constructing the "Celebrity Capital" of Presidential Candidates Online.

Comparative Analysis of 2012 French Presidential Campaign Websites

\title{
Anaïs Theviot
}

\section{(2) OpenEdition}

\section{Journals}

Édition électronique

URL : http://journals.openedition.org/edc/8918

DOI : $10.4000 /$ edc. 8918

ISSN : 2101-0366

Éditeur

Université de Lille

\section{Édition imprimée}

Date de publication : 1 juin 2019

Pagination : 191-208

ISBN : $978-2-917562-21-5$

ISSN : $1270-6841$

\section{Référence électronique}

Anaïs Theviot, « Co-construction du « capital célébrité » du présidentiable en ligne. Analyse comparative des sites de campagne en 2012 », Études de communication [En ligne], 52 | 2019, mis en ligne le 01 janvier 2021, consulté le 21 janvier 2021. URL : http://journals.openedition.org/edc/8918 ; DOI : https://doi.org/10.4000/edc.8918 
Co-construction du " capital célébrité » du présidentiable en ligne. Analyse comparative des sites de campagne en 2012

Co-Constructing the "Celebrity Capital" of Presidential Candidates Online. Comparative Analysis of 2012 French Presidential Campaign Websites

Anaïs Theviot

Université catholique de l'Ouest, ARENES atheviot@uco.fr 
Cet article propose d'appréhender ce que la culture de la célébrité fait à la politique. Autrement dit, il s'agit de questionner l'usage de plus en plus prégnant du marketing en politique et des enjeux d'images, inspirés notamment du "star-system » américain. Certains membres des équipes de campagne $n$ 'hésitent pas à parler d'une entreprise de « peopolisation " des hommes politiques dans une quête constante de visibilité médiatique. Ce travail sur l'image des candidats est particulièrement perceptible sur les sites de campagne car les équipes de communication contrôlent ce dispositif et peuvent donc l'orienter, l'encadrer comme elles le souhaitent. L'objectif premier de ces plateformes en ligne est de faire connaître leur favori en lui élaborant une stature de présidentiable. L'ambition est donc ici d'analyser les stratégies (et leurs limites) de construction de la " célébrité " du présidentiable, en étudiant les homepages des sites de campagne, ainsi que les pages Facebook des deux principaux candidats à l'élection présidentielle française de 2012.

Mots-clés : célébrité, élection présidentielle, site internet, partis politiques, facebook, communication politique.
This article explores the impact of celebrity culture on politics. Our objective is to question the increasing use of marketing and image construction in politics, as inspired by the American star system. Campaign staff members sometimes refer to the "peopolisation" ("celebritisation") of politicians as a necessary means to obtain media visibility. The shaping of a candidate's image is particularly visible on campaign websites which are controlled by communication teams. Online platforms seek to elevate their candidate to the stature of a potential presidential candidate. We conduct analysis of the strategies (and limitations) of online political celebrity image creation, by studying the campaign website home pages and Facebook pages of the two main candidates in the 2012 French presidential elections

Keywords: celebrity, presidential election, website, political parties, facebook, political communication. 
La personnalisation du pouvoir, couplée à sa médiatisation, n'est pas nouvelle. En 1960, A. Mabileau soulignait déjà les effets de la télévision sur l'accentuation de la personnalisation du pouvoir du président (Mabileau, 1960).

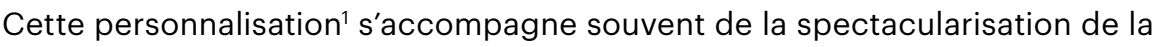
vie politique, accrue par une compétition médiatique propice à des ressorts sensationnalistes (Silke et Maier, 2010). Cela s'avère encore plus prégnant lors des campagnes électorales qui semblent s'apparenter désormais à des campagnes promotionnelles. Leur hyperpersonnalisation amène les équipes de campagne à utiliser les mêmes modes de fonctionnement que des publicitaires ou des agents chargés de promouvoir leurs vedettes. En 1987, I. Rein, P. Kotler et M. Stoller (2006) avaient explicité les méthodes employées pour se gérer soi-même comme une " marque personnelle et professionnelle » et atteindre ainsi une " haute visibilité ». Ces méthodes issues de techniques marketing sont réutilisées à des fins électorales par les candidats qui cherchent à augmenter leur visibilité et ainsi leur légitimité. II s'agit de construire l'image adaptée et attendue du présidentiable à travers un travail de mise en récit et de storytelling (Clodong et Chétochine, 2009), particulièrement visible en ligne (Eyries, 2013). En effet, les équipes de campagne maîtrisent totalement ce qui est mis en ligne sur les supports numériques du candidat (son site de campagne, son blog, sa page Facebook, etc.). Cela permet de contrôler le message diffusé sans passer par I'intermédiaire de journalistes. A. Klein définit les homepages ${ }^{2}$ comme des " dispositifs de mise en forme de soi », des " aire[s] de référence identitaires " (Klein, 2001, 69). Toutefois, les équipes de campagne ne contrôlent pas la suite donnée à leur création, c'est-à-dire les commentaires portant sur leurs sites de campagne, proposés par les journalistes, mais aussi désormais par les citoyens - notamment les militants et sympathisants. Créer son propre support en ligne ne veut pas dire qu'il ne donnera pas lieu à une critique négative sur d'autres espaces numériques. Le contrôle s'avère donc limité vu la multiplicité des lieux d'expression sur la Toile et il s'agit bien plutôt d'une co-construction du « capital célébrité ».

Les travaux anglo-saxons sur les celebrity politics ont déjà bien défriché les interférences entre champ politique et culture populaire, entre médiatisation et sensationnel dans le domaine politique (Street, 2004) : " la vedettisation du politique, baptisée dans les pays anglo-saxons par "celebrity politics" s'apparent à un double mouvement : des responsables politiques vers le monde du spectacle et des célébrités vers la politique " (Dakhlia, 2008, 12). Nous souhaitons étudier la première tendance à travers le cas de la campagne pour l'élection présidentielle de 2012, en portant le regard sur la construction de l'image en

1 Dans la littérature, la notion de personnalisation est utilisée pour référer aux transformations des pratiques politiques qui placent sur le devant de la scène (médiatique) les personnalités politiques, notamment en faisant référence à leur vie privée.

2 Il s'agit des pages d'accueil des sites internet. 
ligne des présidentiables français. Avec la montée en puissance du numérique en campagne électorale, il apparaît intéressant de porter la focale sur les enjeux de la médiatisation en ligne du politique dans ce questionnement sur la construction de l'image du politicien et de sa "célébrité ». En effet, étudier les dispositifs entièrement contrôlés par les équipes de campagne, tels que les sites internet des candidats, permet d'analyser au plus près la « stratégie de célébrité " développée en ligne. Quelles sont ses caractéristiques ? Comment se co-construit leur " capital célébrité » ? Nous retravaillons ici le concept de capital politique, proposé par P. Bourdieu (1981). Ce dernier décrit la société comme un espace de luttes pour l'accumulation de capitaux, inégalement distribués. Ces capitaux orientent l'individu dans l'espace social. II distingue différents types de capitaux, dont le capital politique qui est soit personnel (la notoriété du notable par exemple), soit délégué, transféré d'une institution à un individu (le porte-parole). Le capital célébrité serait ainsi une déclinaison du capital politique lorsque la notoriété serait construite et exacerbée, visant une quête (de pouvoir). Ce capital s'acquiert dans l'espace public et peut se reconvertir dans le champ politique.

Notre enquête porte sur l'analyse de contenu des pages d'accueil des sites de campagne pour l'élection présidentielle de 2012 des candidats du Parti socialiste (PS) et de I'Union pour un Mouvement populaire (UMP), de leurs biographies mises en ligne sur cette plateforme et de la Timeline ${ }^{3}$ du compte Facebook de N. Sarkozy4. L'analyse du design des homepages des sites de campagne de I'UMP et du PS retranscrit des stratégies distinctes, liées au positionnement des candidats. Comme il s'agit de la première perception du site par l'internaute ${ }^{5}$, cette page d'accueil est particulièrement travaillée et pensée en amont. Nous avons choisi, concernant les pages d'accueil des sites de campagne, de focaliser notre analyse sur les images fixes et animées présentes en ligne. Comme l'indique J. Dakhlia $(2011,176)$, « le récit construit autour des

3 Nouveau format disponible de mise en récit de soi sur Facebook : cela s'apparente à un journal intime partant de la naissance du titulaire du compte jusqu'à aujourd'hui.

4 N. Sarkozy a été le premier en 2012 à mettre en place une Timeline, nouveauté alors proposée par Facebook. Son concurrent, F. Hollande, n'a pas créé de Timeline.

5 Il faut toutefois relativiser la portée des sites de campagne des candidats qui sont essentiellement consultés par des sympathisants. L'enquête quantitative collective, menée à la sortie des urnes aux premier et deuxième tours de la primaire de la droite et du centre dans sept sites correspondant à des bureaux primaires, montre qu'internet ne constitue qu'une source d'information marginale pour les électeurs. Ainsi, seuls $22 \%$ des enquêtés ont consulté, au cours de la campagne, le site internet de l'un des candidats, seuls $15 \%$ ont été contactés par mail par une équipe de campagne, seuls $8 \%$ ont déjà « liké » sur Facebook la page d'un candidat et seulement $2 \%$ ont envoyé un message à un candidat sur Twitter (Gouard et al., 2017). Les sites de campagne n'apparaissent pas comme la première source d'information des Français et s'avèrent davantage consultés par les sympathisants, les militants et les journalistes. 
célébrités repose sur un socle visuel "; c'est donc ce socle visuel en ligne qui est ici analysé, complété par le récit biographique présent sur leurs sites de campagne. Enfin, pour ne pas décontextualiser l'analyse de contenu et saisir les intentions des concepteurs, l'enquête s'est vue enrichie d'une cinquantaine d'entretiens semi-directifs réalisés avec les professionnels de la communication numérique des équipes de campagne du PS et de I'UMP en 2007 et 2012. Les premiers entretiens avec les créateurs et les gestionnaires des outils numériques s'apparentent davantage à une recherche d'informations et de justifications des pratiques observées en ligne. Au cours de l'enquête, les entretiens ont été retravaillés et approfondis afin de saisir les cultures partisanes retranscrites à travers les outils numériques et les stratégies de célébrité adoptées en ligne.

Nous verrons que la mise en scène en ligne de ces personnalités politiques varie en fonction de leur capital célébrité. Dans le cas de la campagne pour l'élection présidentielle de 2012, F. Hollande, le challenger a besoin de se faire connaître et reconnaître en tant que présidentiable (appelé en interne « la stratégie de la présidentialisation $\left.{ }^{6} »\right)$, tandis que le candidat sortant souhaite s'imposer comme seule personnalité légitime du fait de son expérience. Malgré ces positionnements différents, ces deux hommes politiques manient en ligne, comme les célébrités, le registre du proche et du lointain pour accroître leur popularité. Enfin, l'étude de la Timeline du compte Facebook de N. Sarkozy soulignera les difficultés pour les professionnels du numérique à raconter avec habileté une histoire aux journalistes et aux électeurs, sans être accusé de manipulation et parodié sur les réseaux sociaux. La construction du capital célébrité est en fait une co-construction où les journalistes, et à présent les citoyens - notamment les adhérents et sympathisants -, jouent un rôle majeur.

1.

\section{Augmenter sa visibilité en ligne : un positionnement distinct entre challenger et président-candidat}

Cette partie se centre sur l'analyse du contenu des pages d'accueil et des biographies (présentes dans des onglets et non en homepage) des sites de campagnes des candidats PS et UMP. Étudier comment se racontent en ligne les candidats dans leur rubrique biographique met au jour les logiques de construction stratégique de leur identité et permet ainsi de saisir la première étape de la construction du capital célébrité, c'est-à-dire celle maîtrisée par les équipes de campagne. L'attention est portée sur l'autopromotion comme forme

6 «À l'origine de la campagne, il y avait un candidat qui était assez peu connu à qui il fallait donner cette stature présidentielle. Ce que l'on appelait la stratégie de présidentialisation. "Claire, responsable du porte-à-porte, puis coordinatrice de l'équipe numérique de F. Hollande. Entretien du 4 juin 2012 
de persuasion afin d'analyser quelle « image l'énonciateur construit de lui-même dans son discours " (Amossy, 2010, 1). L'affrontement d'un président-candidat avec un challenger se retrouve en effet dans les dynamiques de présentation des candidats en ligne. Le président-candidat s'appuie ainsi sur son expérience passée en tant que chef d'État et cherche à maintenir l'avance prise sur ses challengers, notamment en termes de visibilité auprès des électeurs français et sur le plan international. En tant que président sortant, N. Sarkozy s'impose facilement comme leader en 2012 au sein de son parti politique (aucune primaire n'est instaurée) et peut centrer toute l'attention médiatique sur sa personne. L'étude du design de la page de son site, au moment de son lancement mi-février 2012 (cf. figure 1), souligne une mise en avant du candidat avec, sur la même page, trois photographies de ce dernier.

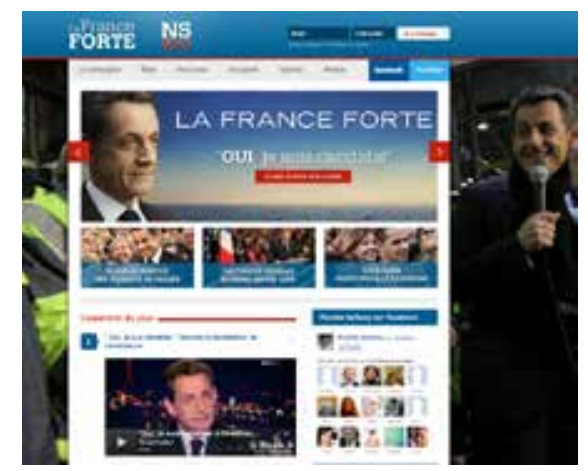

Figure 1 : Capture d'écran de la page d'accueil de franceforte.fr, le 16 février 2012

La première reprend l'image de son affiche de campagne et se place comme une bannière du site que l'on peut faire dérouler. La deuxième se trouve en arrière-plan et se veut donc plus statique. Enfin la troisième propose une vidéo du président annonçant sa candidature lors du journal télévisé de TF1, le 15 février 2012. À cela s'ajoutent trois autres photographies, en format plus réduit, du candidat de I'UMP'. En somme, sur cette page d'accueil, six photographies du président-candidat sont répertoriées : I'hyperpersonnalisation autour de la candidature de N. Sarkozy est ici très lisible. La photographie choisie pour son affiche de campagne, et reprise sur son site internet, cherche à le mettre en scène comme le " capitaine d'un bateau " pouvant sortir les Français de la crise. Il a un air serein et posé qui inspire confiance, à l'opposé des propriétés qu'on lui attribue généralement (nerveux, stressé, pressé, hyperactif, etc.). La

7 La première image réduite de N. Sarkozy le montre souriant au milieu de citoyens afin d'illustrer la rubrique « 30 ans au service des Français ». Dans la seconde, il est représenté en train de prendre la parole lors d'un meeting. Enfin, la troisième image reprend la photographie présente sur le profil de son compte Facebook. 
sobriété de cette affiche - regard fixe vers un horizon dominé par le bleu de la mer et du ciel - éloigne le candidat du style "bling bling " qui lui est accolé depuis son élection en 2007, à la suite notamment de la soirée au Fouquet's et aux vacances sur le yacht de V. Bolloré. En outre, sur cette photographie, les rides du président sont laissées bien visibles, ainsi que ses cheveux blancs (qui ressortent encore davantage avec la couleur choisie pour le slogan, lui-même en blanc). Cette chevelure poivre et sel a été largement commentée dans les médias ${ }^{8}$ et semble vouloir mettre en avant l'expérience du président-candidat. Elle lui apporte également un élément de séduction, proche des stars hollywoodiennes (Dyer, 2004) qui savent jouer avec leur âge à l'instar de G. Clooney.

Au-delà des images, le site internet de campagne de N. Sarkozy accorde, dans son contenu, une place majeure à la promotion du bilan, avant même le programme. En tant que président-candidat, il se doit de mettre en avant les actions effectuées pendant sa mandature. Sur la page d'accueil, deux liens pointent vers le bilan de N. Sarkozy ${ }^{9}$. Cette rubrique donne accès à vingt-huit thèmes où est valorisée l'action du président sortant. On y retrouve des thématiques chères à l'électorat traditionnel de I'UMP, telles que : « agriculture et pêche " ou «travail et industrie ". D'autres rubriques s'avèrent plus ancrées dans la stratégie de campagne du candidat de I'UMP :

"La France forte dans le monde " ou " Face à la crise ». Chaque thème ouvre sur un résumé de l'action du président sortant, accompagné d'une photo de celui-ci le mettant en scène sur le terrain. Sur le site internet de campagne, le bilan de N. Sarkozy est donc fortement mis en avant : on a essayé de mettre l'accent sur les contenus, sur les programmes, les chiffrages, les bilans (Responsable de la promotion du bilan de N. Sarkozy au sein de l'équipe web du QG. Entretien du 1er février 2013).

Des campagnes de mailings ("Ce qui a changé pour vous dans les cinq dernières années... »), complétées par un travail sur les réseaux sociaux centré sur cette valorisation du bilan, ont été effectuées afin d'élargir l'échantillon des personnes touchées.

À l'inverse, sur son site de campagne, F. Hollande ne peut présenter de bilan, n'ayant pas exercé cette fonction auparavant, mais propose, tout de même, le bilan de sa carrière politique afin de légitimer sa candidature. La primaire socialiste de 2011 a participé à la construction de la célébrité de F. Hollande.

8 "J'ai des rides, des cheveux blancs, un regard fatigué car je passe mes jours et mes nuits à travailler pour vous", commentaire d'un internaute (posté le 16 février 2012) à l'article du Nouvel observateur (12 septembre 2011). "Sarkozy 2012 : un plagiat de la Force tranquille? » [en ligne], http://leplus.nouvelobs. com/contribution/191781-sarkozy-2012-un-plagiat-de-la-force-tranquille.html (consulté le 2 octobre 2013).

9 Un onglet « bilan » est mis en avant dans l'affichage en haut à gauche sur la page d'accueil et est rappelé également en bas à gauche dans la rubrique "I'action de Nicolas Sarkozy depuis 2007 ». 
Mais les citoyens dits " ordinaires ${ }^{10}$ n'ont pas forcément connaissance de sa carrière politique; d'autant plus qu'à partir de 2008, à la fin de son mandat en tant que premier secrétaire du PS, la visibilité médiatique de F. Hollande s'avère très limitée (contrairement à son adversaire). Son influence sur les directives du parti s'est également considérablement réduite et les partisans de son courant se sont progressivement amoindris. De 2008 à 2012, il exerce les fonctions de député de la première circonscription de la Corrèze et préside son Conseil général, mais il reste dans l'ombre de l'actualité politique nationale. Peu de socialistes avaient parié sur sa victoire aux primaires en 2011, avec le spectre de la candidature plébiscitée de D. Strauss-Khan. Comme le souligne un membre de son équipe web, F. Hollande se trouvait donc assez isolé, même sur la Toile : "Les gens qui étaient pour François Hollande, il n’y en avait pas beaucoup sur Twitter. Donc on a vite commencé à se connaître. Il y avait beaucoup d'aubryistes parce que c'est le PS. II y avait beaucoup de strauss-kahnniens. II ne faut pas oublier qu'à l'époque il bouffait tout. Et pour Hollande, on devait être dix à vingt » (Entretien du 2 mars 2012).

II s'agit donc pour l'équipe de campagne de F. Hollande de profiter de la vague médiatique des Primaires pour imposer son candidat comme le seul pouvant affronter N. Sarkozy au second tour de l'élection présidentielle. L'ambition est à la fois d'orienter le cadrage médiatique pour mettre en scène un duel Hollande/Sarkozy et d'amener les Français à une certaine vision du candidat Hollande. Ainsi, le site de campagne de F. Hollande présente un long texte où le candidat se raconte à la première personne, en faisant preuve d'un certain lyrisme : « [de mes parents] j'ai appris l'attention que l'on doit porter aux autres »; "j'ai découvert la passion de l'engagement militant dans le syndicalisme étudiant "; " comment être le président des Français sans les aimer, sans connaître leur histoire, sans entendre la respiration profonde du pays ? $"^{11}$. L'emploi du pronom personnel « je » n'est pas anodin, surtout dans la communication d'un candidat à gauche de l'échiquier politique où le collectif est souvent mis en avant par l'emploi du « nous ». Dans cette longue biographie, on retrouve les traits caractéristiques des leaders politiques, repérés par A. Bittner (2011), à la suite de sa méta-analyse des études électorales : le leadership, I'honnêteté, la fiabilité, la compassion, la connaissance des dossiers, l'intelligence et le souci du bien-être des citoyens.

Ce choix du « je » est aussi adopté pour présenter son projet en ligne : " je veux redresser la France », " je veux rétablir la justice », " je veux redonner

10 Ordinaire ne s'oppose pas à son corollaire " extraordinaire ", il s'agit de se référer au " commun ». Cette expression renvoie au sentiment de compétence et aux ressources inégalement distribuées ce qui amène à distinguer des profils différents, se confrontant à la figure de l'expert, du professionnel, du membre de l'organisation.

11 Extrait du site internet de campagne de F. Hollande, françoishollande.fr (consulté le 8 avril 2012). 
espoir aux nouvelles générations » et « je veux une République exemplaire et une France qui fasse entendre sa voix ». Le pronom personnel " je », employé par les candidats sur leurs sites de campagne, atteste de la personnalisation du scrutin afin de gagner en capital célébrité. L'un des objectifs du site de campagne de F. Hollande est de présenter le candidat socialiste, en accordant son portrait aux normes attendues du présidentiable. Comme le souligne le rédacteur en chef du site françoishollande.fr, " l'objectif, c'était d'abord de couvrir la campagne, d'accompagner François Hollande dans sa campagne, de donner à voir ce qu'il faisait. Ensuite, c'était de vendre le projet, de le faire connaître, de le décliner, de l'expliquer. » (Entretien du 2 janvier 2013).

N. Sarkozy et F. Hollande ont le même objectif : paraître le plus légitime dans le rôle de président de la République, notamment en augmentant leur capital célébrité pour s'imposer comme seul légitime. L'un s'appuie sur le capital célébrité déjà éprouvé et construit en tant qu'ancien président tandis que l'autre se réfère à un capital célébrité en construction et dans une démarche d'ascension, en mettant en avant sa carrière politique qui l'aurait destinée à ce rôle et en s'appuyant ainsi sur son capital politique.

\section{2.}

\section{Un point commun aux hommes politiques et aux célébrités : mixer le registre du proche et du lointain}

En 1985, Richard Schickel propose une analyse des célébrités comme des "étrangers intimes " [intimate strangers] ${ }^{12}$, soulignant les frontières flottantes entre vie privée et vie publique. De cette façon, la star ne paraît pas éloignée de ses fans qui partagent une partie de son intimité dévoilée, tout en continuant à susciter l'admiration en préservant le mystère. Les hommes politiques qui cherchent à s'imposer comme les futurs élus jouent aussi avec les registres du proche et du lointain qui s'entremêlent en travaillant une rhétorique de la proximité (chat en ligne, serrer des mains, président " normal »), tout en apparaissant comme hors norme, plus compétents, en surplomb de l'électeur " ordinaire ". Les médias participent à ce registre du proche en multipliant les couvertures de presse avec des hommes politiques à la Une, voire en réalisant des reportages révélant une partie de leur vie privée. Ce jeu commun aux deux principaux candidats à l'élection présidentielle en 2012 se retrouve en ligne dans les contenus et images mises en avant sur leur site de campagne. L'objectif est de renforcer le capital célébrité du candidat afin qu'il devienne familier à l'électeur (apport affectif, registre de la proximité), tout en lui adossant un statut 
de présidentiable (registre du lointain, hauteur, surplomb). Pour répondre au premier registre de proximité, sur le site de campagne de F. Hollande, il s'agit de "faire vrai ». Les images choisies participent donc à construire l'image d'une personne proche des citoyens. Elles ne sont pas sélectionnées dans une esthétisation du numérique, mais cherchent plutôt à paraître proches du terrain. La responsable du compte Twitter de F. Hollande en 2012 souligne bien que son choix de photographie se porte d'abord sur celle qui fait la plus " vraie », avant celle qui est la plus belle :

Il y a plein de fois où même des journalistes qui m'appelaient en me disant : "Quand même t'es chiée de ne pas utiliser Instagram et tout ». Je leur disais : "Non, mais c'est non ». Une campagne, c'est pas un truc joli, c'est pas un truc avec des jolies couleurs où... Le nombre de fois où j'ai pris des photos d'Hollande immondes et genre les gens m'appelaient. Mais non, c'est la vraie vie. Ce n'est pas une star de cinéma, il n'est pas mannequin, il est candidat à l'élection présidentielle, il est dans la boue. Il pleut, il est avec des agriculteurs. Il est moche. Les agriculteurs sont moches. Eh ben, c'est le cas de ce mardi matin. Non mais tu vois ce que je veux dire... II n'y a pas de... Enfin, l'esthétisation d'une campagne, je trouve ça bidon (Entretien du 11 octobre 2012).

Cette envie de "faire vrai » se retrouve aussi chez les « people » : beaucoup d'actrices américaines (Cameron Diaz, Jessica Alba...), chanteuses et mannequins s'affichent sur Instagram au naturel à grand renfort de hashtags " nude ", " no filter " et " no make-up ». En réaction aux images filtrées des campagnes de publicité et des photos postées sur les réseaux sociaux, ces célébrités revendiquent un retour à une certaine authenticité. La mode du « vrai » pour paraître proche des "fans » ou des électeurs apparaît comme un moyen pour augmenter sa popularité et accroitre ainsi son capital célébrité.

Lors de sa première candidature à l'élection présidentielle de 2007, N. Sakozy avait aussi joué sur le registre de la proximité. Cette stratégie de communication s'inscrit dans l'optique globale de renouvellement, ici le « renouvellement des registres de grandeur politique » (Le Bart, 2005, 27 - souligné par l'auteur). Dans la quête de la présidence de la République par N. Sarkozy en 2007, C. Le Bart s'interroge sur la possibilité d'avoir recours, pour les présidentiables, au registre de la proximité - ce poste étant censé se fonder sur une symbolique de la grandeur, de la hauteur et du surplomb ${ }^{13}$, alors incompatible avec la rhétorique du proche. C. Le Bart conclut que «le moment de la campagne se prête idéalement au déploiement de cette symbolique [de la proximité], tout candidat se devant d'apparaître à l'écoute sinon en contact rapproché 
avec les citoyens » (Le Bart, 2009, 42). Autrement dit, le présidentiable, encore plus que le président lui-même, invoque ce registre pour séduire ses possibles électeurs.

Ce travail, de plus en plus professionnel, sur l'image des candidats, inspiré des techniques marketing et de l'attrait pour le star-system américain, est assumé par une partie des équipes de campagne qui n'hésitent pas à parler par exemple d'une entreprise de " peopolisation » (Dakhlia et Lhérault, 2008) du dispositif porte-à-porte pour attirer le regard médiatique ${ }^{14}$ :

J'ai « peopolisé » le porte-à-porte. Je maintiens mon point de vue qu'il faut que ce soit sexy de faire du porte-à-porte et pas juste laborieux. Et la « peopolisation » du porte-à-porte... Au début, les politiques m'ont dit : « mais ça va pas! ». Dieu merci, il y avait Ségolène... Et ça, c'est super parce que ça montre un engagement, ça montre le boulot (Membre de l'équipe communication de F. Hollande. Entretien du 15 septembre 2012).

Avec le cas du porte-à-porte, il s'agit encore une fois de paraître proche de l'électeur, en allant le rencontrer à son domicile. Cette proximité construite est mise en scène et les équipes de campagne n'hésitent pas à largement communiquer sur ces événements pour qu'ils soient repris dans les médias. L'ambition des équipes de campagne est bel et bien de participer à la construction du capital célébrité des candidats en utilisant les mêmes techniques marketing que pour vendre un produit à l'électeur-consommateur. Mais en usant des techniques marketing, les communicants se confrontent aux risques qu'elles comportent : une réappropriation du message par les internautes qui peuvent alors le détourner et contribuer à discréditer le candidat.

3.

\section{Les risques du storytelling en ligne: le cas de la Timeline de N. Sarkozy sur Facebook}

Suite à l'annonce officielle de la candidature de N. Sarkozy à l'élection présidentielle à la mi-février, au journal télévisé de 20 heures sur TF1, I'UMP a mis en ligne une fan page Facebook dédiée à la trajectoire de N. Sarkozy. II s'agit alors de paraître innovant en s'emparant d'un nouveau format disponible

14 S. Royal a été filmée par les équipes de communication de F. Hollande allant frapper aux portes lors de la campagne de 2012. D'autres figures politiques socialistes ont ensuite " joué le jeu », telles que N. Vallaud-Belkacem, A. Filippetti, J. Lang, etc. Cette mise en scène du politique-star allant frapper aux portes des citoyens " ordinaires " a contribué au succès médiatique du volet porte-à-porte. 
sur Facebook : la Timeline ou journal. Comme le précise Facebook, il s'agit d'un nouveau type de profil, plus proche d'une biographie ${ }^{15}$ :

Alors la Timeline, c'est un changement qui était d'abord une fonctionnalité nouvelle qu'a proposé Facebook. Ce n'était pas disponible avant l'automne dernier je crois, ou décembre. Donc la question était comment utiliser cette nouvelle fonctionnalité. Et à partir de fin mars je crois, c'est devenu une obligation. Toutes les pages ont basculé. Donc c'est important pour nous aussi de voir quel parti on pouvait tirer de cette affaire-là et d'en faire aussi un petit enjeu com' (Directeur du web au siège du PS, à Solférino. Entretien du 21 mai 2012).

Sur Facebook, la Timeline de N. Sarkozy se centre uniquement sur le parcours du candidat et ses rencontres politiques (l'objectif est ici de rendre visible son capital politique), mais aussi ses interactions avec des vedettes notamment internationales (logique de transfert de popularité). Son parcours politique est ainsi retracé avec des documents multimédias (photos, vidéos, extraits de discours, etc.). La chronologie débute à la naissance du président sortant jusqu'à son annonce de candidature à l'élection présidentielle de $2012^{16}$. Cette Timeline a été construite pour valoriser le bilan de N. Sarkozy. Entre le listing des grandes réformes (la suppression des droits de succession, l'instauration des peines planchers pour lutter contre la récidive, l'autonomie des universités, l'accompagnement éducatif pour "les orphelins de $16 \mathrm{~h}$ » et la réforme des retraites sont notamment évoqués), la mise en avant du rôle international de la France (l'intervention militaire en Libye, la présidence de I'Union européenne, la lutte contre la crise économique) et les nombreux déplacements du président, le profil propose un bilan quasi complet des cinq dernières années. Mais seuls les éléments positifs sont mentionnés. En effet, ont été écartées de cette chronologie la perte du triple A ou les défaites électorales subies par I'UMP durant cinq ans (municipales de 2008, régionales en 2010, sénatoriales et cantonales en 2011). Comme le souligne J. Gerstlé, la capacité à orienter l'attention publique sur tel ou tel objet/thématique fait partie des stratégies habituelles des professionnels de la communication politique : «il faut mentionner le pouvoir qu'ont les moyens d'information d'attirer l'attention publique sur certains objets privilégiés dans l'environnement. Ce faisant, ils laissent dans l'ombre toute une série d'autres objets » (Gerstlé, 2012, 78). Ainsi, laisser dans l'ombre les évènements négatifs du quinquennat de N. Sarkozy

15 Une chronologie apparaît sur la droite de l'écran afin de répertorier dans le temps les événements (anciennes publications, photos, vidéos...) de la vie du détenteur du compte Facebook.

16 On retrouve ainsi N. Sarkozy lors de son premier passage à la télévision en 1975 ou lors de son premier discours à Nice aux côtés de J. Chirac en 1976. À partir du discours d'investiture de janvier 2007, la Timeline de N. Sarkozy s'enrichit de détails afin de retracer sa campagne, ses meetings et ses rencontres, avant de s'attaquer au point le plus important de ce profil : la valorisation du quinquennat. 
relève d'une stratégie de communication volontaire afin que les internautes « oublient » ces échecs.

Utiliser ce nouveau dispositif de la Timeline est aussi un moyen pour l'équipe de communication de N. Sarkozy de faire parler de leur candidat, de donner un sujet "prêt à médiatiser " aux journalistes. Dans son cas d'étude portant sur les journalistes parlementaires au Québec, J. Charron (1994) souligne que les conseillers en communication des hommes politiques ont bien décelé les routines et les contraintes professionnelles des journalistes. Cette grille de lecture leur permet d'anticiper les comportements et les attentes des reporters afin de passer l'information qu'ils souhaitent diffuser. Toutefois, dans le cas de la Timeline de N. Sarkozy, les omissions quasi-caricaturales se sont avérées contre-productives médiatiquement. De nombreux journalistes ont choisi de cadrer leur article, portant sur ce dispositif, sur la reconstruction du quinquennat : " biographie en forme de panégyrique facebookien, et ses silences pudiques sur tout ce qui, dans I'histoire de l'actuel président de la République, pourrait s'avérer contre-productif pour un homme en campagne ${ }^{17}$ ". Les commentaires des internautes ont rapidement aussi été critiques et le travail de mise en scène de la carrière de N. Sarkozy a ainsi été décortiqué pour laisser apparaître les stratégies de construction sélective. Des parodies humoristiques ont vite été partagées sur Facebook et sont devenues très virales. Le site lavraietimeline.fr (image 2) propose ainsi une refonte complète de la page Facebook du candidat UMP, en réintroduisant certains éléments tels que la rencontre avec M. Kadhafi, le rapatriement de son fils P. Sarkozy ou encore ses mauvais scores dans les sondages ${ }^{18}$.

17 Le Nouvel Observateur (6 mars 2012). "Sarkozy, sa campagne en forme de Timeline Facebook » [en ligne], http://tempsreel.nouvelobs.com/presidentielle-la-bataille-du-web/20120306.OBS3018/sarkozy-sa-campagne-enforme-de-Timeline-facebook.html (consulté le 6 août 2013).

18 Le texte de la bannière donne le ton : "Je n'éprouve depuis le plus jeune âge aucun scrupule à dire tout et son contraire pour servir les riches et les puissants d'une grande, vieille et belle nation : la France [propos attribué à N. Sarkozy] ». Message présent sur la page d'accueil de la version parodique de la Timeline de N. Sarkozy. 


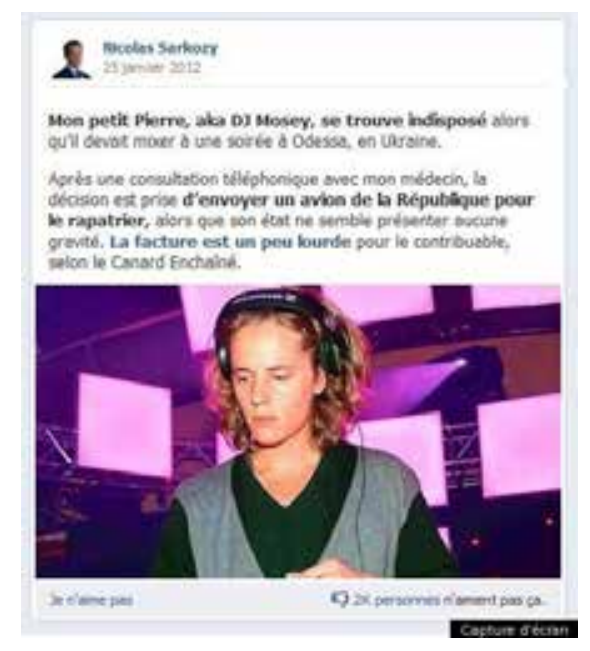

Figure 2 : Capture d'écran du site parodique de la Timeline de N. Sarkozy, lavraietimeline.fr

On le voit bien avec le cas de la Timeline de N. Sarkozy, le capital célébrité s'avère co-construit en ligne à la fois par l'équipe de communication qui cherche à donner une image positive du candidat, mais aussi par les journalistes qui investissent une mission critique, et plus largement les internautes qui s'amusent de la communication officielle. Lors de son analyse de la campagne pour l'élection présidentielle de 2007, C. Lemieux soulignait que « le personnel politique n'hésite plus à devancer les attentes des journalistes sur ce terrain en s'adaptant aux formats que ces derniers cherchent à remplir " (Lemieux, $2010,76)$. Il évoquait le jeu des « petites phrases » qui permet de rentrer dans le format court des reportages du journal télévisé. En ligne, on retrouve cette adaptation des formats, avec une écriture très simplifiée, des intitulés incisifs et la multiplication des visuels. Ce règne de l'image en ligne induit une simplification du message politique et invite à esthétiser le politique.

\section{Conclusion}

En période de campagne électorale, les candidats doivent développer un style singulier pour se différencier de leur adversaire. Le travail sur l'image des candidats est particulièrement perceptible sur les sites de campagne car les équipes de communication contrôlent ce dispositif et peuvent donc l'orienter, l'encadrer comme elles le souhaitent. L'objectif premier de ces plateformes en ligne est de faire connaître leur favori en lui élaborant une stature de présidentiable et en participant ainsi à la construction de son capital célébrité. Les candidats à l'élection présidentielle investissent désormais de manière massive les dispositifs en ligne pour paraître modernes et innovants (Theviot, 
2018), mais n'en comprennent pas toujours les codes. Créer des plateformes en ligne permet en effet de contrôler le contenu sur ces outils, mais n'empêche pas qu'ils soient contournés, décriés et ridiculisés sur d'autres espaces numériques, notamment sur les réseaux sociaux.

Comme dans les classiques théories marketing pour un produit ou une marque, l'enjeu essentiel du capital célébrité est bien de faire parler de soi, d'accroître sa visibilité. Peu importe si l'on parle de vous de manière négative, le plus important étant que l'on parle de vous. Le capital politique est "fondé sur la croyance ", avec pour enjeu essentiel "d'accumuler le crédit et d'éviter le discrédit " (Bourdieu, 1981, 24) tandis que le capital célébrité est fondé sur la quête accrue et tous azimuts de la notoriété, qu'elle soit positive ou négative : "Mediated visbility could also be a trap "19 (Thompson, 2000b, 41). La « peopolisation subie » (Dakhlia, 2010, 16) participe ainsi à la construction de ce capital célébrité, tout comme les détournements péjoratifs (ou du moins tournant en ridicule) des éléments de communication officielle du candidat.

Le capital célébrité est ainsi co-construit par les équipes de campagne, les journalistes, les militants et tous ceux qui participent à la circulation en ligne de contenu politique (ou non-politique) sur le candidat. Cette co-construction a longtemps été minorée en France : la culture politique française a contribué à limiter la montée en puissance de ce capital célébrité dans le champ politique, notamment dans son versant négatif (tradition française d'opacité de la vie privée des politiciens, législation stricte autour des publicités politiques, etc.). Toutefois, la campagne pour l'élection présidentielle française de 2017 laisse à penser que le capital célébrité des politiciens est en train de se reconfigurer avec la montée en puissance des fausses informations circulant massivement en ligne. Le numérique semble en effet introduire des changements dans la co-construction des stratégies de célébrité, au sens où les militants, et plus largement les citoyens, peuvent participer de manière prégnante à la quête de visibilité des candidats en réagissant, commentant ou créant des contenus en ligne, facilement partageables sur les réseaux sociaux de manière massive, instantanée et quasi-automatique. 
Bibliographie

Amossy R. (2010). La présentation de soi. Éthos et identité verbale, Paris, Presses universitaires de France.

Bittner A. (2011). Platform or Personality? The Role of Party Leaders in Elections, Oxford, Oxford University Press.

Bourdieu P. (1981). " La représentation politique ». In Actes de la recherche en sciences sociales, vol. 36-37, p. 3-24.

Charron J. (1994). La production de l'actualité. Une analyse stratégique des relations entre la presse parlementaire et les autorités politiques au Québec, Québec, Boréal.

Clodong O., Chétochine G. (2009). Le storytelling en action. Transformer un politique, un cadre d'entreprise ou un baril de lessive en héros de saga ! Paris, Groupe Eyrolles.

Dakhlia J. (2008). Politique people, Paris, Bréal.

Dakhlia J. (2010). Mythologie de la peopolisation, France, Le cavalier bleu.

Dakhlia J. (2011). « La visibilité people, ennemie de la démocratie? ». In Aubert N. et Haroche C. (dir.), Les tyrannies de la visibilité. Être visible pour exister? Toulouse, ERES, p. 171-190.
Dakhlia J. et Lhérault M. (2008). «Peopolisation et politique ». In Le Temps des médias, vol. 10, n¹, p. 8-12.

Dyer R. (2004 [1979]). « Deuxième partie : La star comme image médiatique ». In Dyer R. (dir.), Le star-système hollywoodien, suivi de Marilyn Monroe et la sexualité, trad. N. Burch. Paris, L'Harmattan, p. 28-67.

Eyries A. (2013). « Twitter et le microstorytelling : un nouveau lieu de légitimation pour les leaders politiques ». In Gallezot G. et Pelissier N. (dir.), Twitter : Un monde en tout petit ? Paris, L'Harmattan, p. 195-208.

Gerstlé J. (2012). « Les effets de la disjonction et de la conjonction entre information et communication électorale ». In Cayrol R. et Charon J.-M. (dir.), Médias, opinions et présidentielles. Paris, Éditions INA, p. 71-78.

Gouard D., Audemard J., Boyadjian J., Marchand-Lagier C., Mathieu R., Olivier L. et Theviot A. (2017). «Les trois électorats de la primaire de la droite et du centre : Mobilisation et production des votes aux limites de l'entre-soi ". In Revue française de science politique, vol. $67, \mathrm{n}^{\circ} 6, \mathrm{p} .1113-1130$.

Klein A. (2001). " Les homepages, nouvelles écritures de soi, nouvelles lectures de l'autre ». In Spirale - Revue de recherches en éducation, vol. 28, p. 67-82.

Le Bart C. (2005). « La proximité selon Raffarin ». In Mots. Les langages du politique, vol. 77, p. 13-28. 
Le Bart C. (2009). « Les présidentiables de 2007 entre proximité et surplomb. Nicolas Sarkozy et Ségolène Royal vus par Libération ». In Mots. Les langages du politique, vol. 89, p. 39-55

Lemieux C. (2010). Un président élu par les médias ? Regard sociologique sur la présidentielle de 2007, Paris, Presses des Mines.

Mabileau A. (1960). «La personnalisation du pouvoir dans les gouvernements démocratiques ". In Revue française de science politique, vol. 10, p. 39-45.

Pirat B. (2002). « En haut et au centre : la prééminence présidentielle ". In Mots. Les langages du politique, vol. 68, p. 59-72.

Rein I. J., Kotler Ph., Stoller M. R. (2006 [1987]). High visibility: transforming your personal and professional brand, New York, McGraw-Hill.

Schickel R. (1985). Intimate strangers: the culture of celebrity, Garden City, Doubleday.

Silke A., Maier M. (2010). «Personalization of Politics. A Critical Review and Agenda of Research ». In Communication Yearbook, vol. 34, p. 212-257.

Street J. (2004). « Celebrity

Politicians : Popular Culture and Political Representation ». In British Journal of Politics \& International Relations, vol. 6, n 4, p. 435-452.
Theviot A. (2018). Faire campagne sur Internet, Villeneuve d'Ascq, Presses universitaires du Septentrion.

Thompson J. B. (2000a). « Transformation de la visibilité ". In Réseaux, vol. 100, p. 187-213.

Thompson J. B. (2000b). Political Scandal. Power and Visibility in the Media Age, Cambridge, Polity Press. 
